\title{
Autonomia e necessidades de saúde na Sistematização da Assistência de Enfermagem no olhar da saúde coletiva
}

\author{
AUTONOMY AND HEALTH NEEDS IN THE SYSTEMATIZATION OF \\ NURSING ASSISTANCE UNDER THE OPTICS OF THE PRIMARY CARE
}

\author{
AUTONOMÍA Y NECESIDADES DE SALUD EN LA SISTEMATIZACIÓN \\ DE LAASISTENCIA DE ENFERMERÍA BAJO LAÓPTICA DE LAATENCIÓN PRIMARIA
}

Débora Gomes Barros', Anna Maria Chiesa²

\section{RESUMO}

Face às mudanças na organização da atenção básica, torna-se necessário refletir sobre as contribuições da Enfermagem no âmbito assistencial. Este artigo teve como objetivo revisar os conceitos de autonomia e necessidades de saúde e suas aplicações nas diferentes propostas de sistematização da assistência de enfermagem. Foi feita uma revisão bibliográfica sobre sistematização da assistência de enfermagem, autonomia e necessidades de saúde nas bases de dados LILACS e BDENF. Os resultados indicaram que a autonomia se refere ao profissional e ao paciente. Quanto ao tema necessidades, verificaram-se as vertentes necessidades biológicas e sociais, que se interceptam com a psicológica, para abranger as necessidades biopsicossociais. Verificou-se que a aplicação dos conceitos analisados não estão presentes em todos os sistemas de classificação de enfermagem, os que mais se aproximam são os projetos de Classificação Internacional das Práticas de Enfermagem (CIPE) e Classificação Internacional das Práticas de Enfermagem em Saúde Coletiva (CIPESC).

\section{DESCRITORES}

Enfermagem em saúde pública. Atenção primária à saúde. Necessidades e demandas de serviços de saúde.

Processos de enfermagem.

\begin{abstract}
Given recent changes in the organization of the primary health care in Brazil, it is necessary to reflect on the contributions of nursing care. This article aims to review the concepts of autonomy and health needs and its applications in different proposals for the systematization of the nursing care. It is a literature review on systematization of the nursing assistance, autonomy and health needs in databases LILACS and BDENF. The most relevant results indicate that autonomy incorporates aspects professional and patient's that are sustained by their respective categories. About needs we found that tracks biological needs and social needs, which intersect with the psychological to cover biopsychosocial needs. It was found that the application of the concepts was not present in classification systems of nursing. However, they were more related to International Classification of Nursing Practice (ICNP) and International Classification of Nursing Practice in Collective Heath (ICNPCH) projects.
\end{abstract}

\section{RESUMEN}

Frente a los cambios en la organización de la atención básica, se torna necesario reflexionar sobre las contribuciones de enfermería en el ámbito asistencial. Este artículo tiene como objetivo revisar los conceptos de autonomía e necesidades de salud, y revisar sus aplicaciones en las diferentes propuestas para sistematización de la asistencia de enfermería. Se realizó una revisión bibliográfica sobre: sistematización de la asistencia de enfermería, autonomía y necesidades de salud, en las bases LILACS y BDENF. Los resultados indican que la autonomía contempla las vertientes profesionales y del paciente. Con respecto al tema necesidades, se verifican las vertientes necesidades biológicas y sociales, que se interceptan con la psicológica para abarcar las necesidades biopsicosociales. Se comprobó que la aplicación de los conceptos analizados, no están presentes en todos los sistemas de clasificación de enfermería, sin embargo, los que más se aproximan son los proyectos de CIPE y CIPESC.
1 Graduanda da Escola
de Enfermagem,
Universidade de São
Paulo (EEUSP).
Membro do Grupo de
Pesquisa Modelos
Tecno-Assistenciais e
a Promoção da
Saúde. Bolsista
PIBIC/CNPq. São
Paulo, SP, Brasil.
debygb@yahoo.com.br
2 Enfermeira. Profes-
sora Associada do
Departamento de
Enfermagem em
Saúde Coletiva,
Escola de Enferma-
gem, Universidade
de São Paulo (EEUSP).
Coordenadora do
Grupo de Pesquisa
Modelos Tecno-Assis-
tenciais e a Promoção
da Saúde. Chefe do
Departamento de En-
fermagem em Saúde
Coletiva, Escola de
Enfermagem, Univer-
sidade de São Paulo
(EEUSP). São Paulo,
SP, Brasil.
amchiesa@usp.br

\section{DESCRIPTORES}

\section{KEY WORDS}

Public health nursing.

Primary health care.

Health services needs

and demand.

Nursing process.
Enfermería en salud publica.

Atención primaria de salud.

Necesidades y demandas de servicios de salud.

Procesos de enfermería. 


\section{INTRODUÇÃO}

A proposta do Programa Saúde da Família (PSF) baseiase, sobretudo, no território adscrito e no processo de vinculação dos profissionais com a população. Tem como uma das atividades estratégicas a visita domiciliária que permite à equipe conhecer os arranjos familiares e problemas de saúde enfrentados. Desse modo, pode-se aproximar das necessidades reais da coletividade como meio de estruturar as ações em saúde.

Depara-se com o surgimento de práticas que possibilitam intervir sobre problemas e seus condicionantes, como: cadastramento que informa as condições de vida e trabalho da população; as visitas domiciliárias que permitem conhecer o indivíduo inserido na família; além dos programas de prevenção e promoção à saúde. Este cenário corrobora para necessidade da enfermagem em saúde coletiva rever o seu processo de sistematização das práticas. Entende-se como Sistematização da Assistência de Enfermagem o processo de identificação de problemas, interpretação e organização de condutas no âmbito do exercício profissional.

\section{OBJETIVOS}

Revisar os conceitos de autonomia e necessidades de saúde e suas aplicações nas diferentes propostas de sistematização da assistência de enfermagem a fim de contribuir com a nova visão de atenção à saúde a partir da assistência de enfermagem.

\section{MÉTODO}

Realizou-se uma revisão bibliográfica sobre sistematização da assistência de enfermagem, autonomia e necessidades de saúde, considerando artigos científicos, teses, dissertações, livros e capítulos de livros dos últimos dez anos, nas bases de dados LILACS (Literatura Latino-Americana e do Caribe em Ciências da Saúde) e BDENF (Base de Dados de Enfermagem), no endereço eletrônico: www.bireme.br e manualmente na Biblioteca Wanda de Aguiar Horta da Escola de Enfermagem da USP. Após a leitura prévia dos resumos dos trabalhos levantados, foram selecionados os pertinentes ao objetivo desta investigação.

Os trabalhos eleitos foram fichados quanto ao assunto, título, ano de publicação, editora, autores, citações relevantes à pesquisa, havendo ao final a produção de resenhas. Posteriormente foram submetidos à análise temática.

\section{RESULTADOS E DISCUSSÃO}

De acordo com os trabalhos analisados, há diversas abordagens sobre as temáticas autonomia, necessidades de saúde e sistematização da assistência de enfermagem.

A análise quantitativa dos temas propostos é apresentada a seguir no Quadro 1.

Quadro 1 - Distribuição bibliográfica analisada sobre autonomia, necessidades de saúde e sistematização da assistência de enfermagem, nas bases de dados LILACS e BDENF no período de agosto a dezembro de 2006 - São Paulo - 2007

\begin{tabular}{|lcc|}
\hline \multicolumn{1}{|c}{ Temas } & LILACS & BDENF \\
\hline Autonomia & 25 & 2 \\
Necessidades de Saúde & 3 & 4 \\
SAE & 8 & 11 \\
\hline Total & 36 & 17 \\
\hline
\end{tabular}

Entre as bases de dados LILACS e BDENF foram levantados no total 53 trabalhos, entre eles, 15 eram repetidos, restando 38 artigos de periódicos para análise. Foram também incluídos na pesquisa 3 capítulos de livros e 1 artigo de periódico levantados manualmente na Biblioteca Wanda de Aguiar Horta da Escola de Enfermagem da USP. Analisaram-se no total 42 trabalhos.
Para a temática autonomia foram encontradas duas vertentes: autonomia profissional (9) e autonomia do paciente (11), que se subdividem em categorias temáticas. Na vertente autonomia profissional encontraram-se as categorias saber próprio da enfermagem e divisão vertical do trabalho e, na vertente autonomia do paciente, encontraram-se as categorias temáticas co-responsabilidade/ mutualidade, flexibilização nas situações de institucionalização e autodeterminação, demonstradas na Figura 1. 


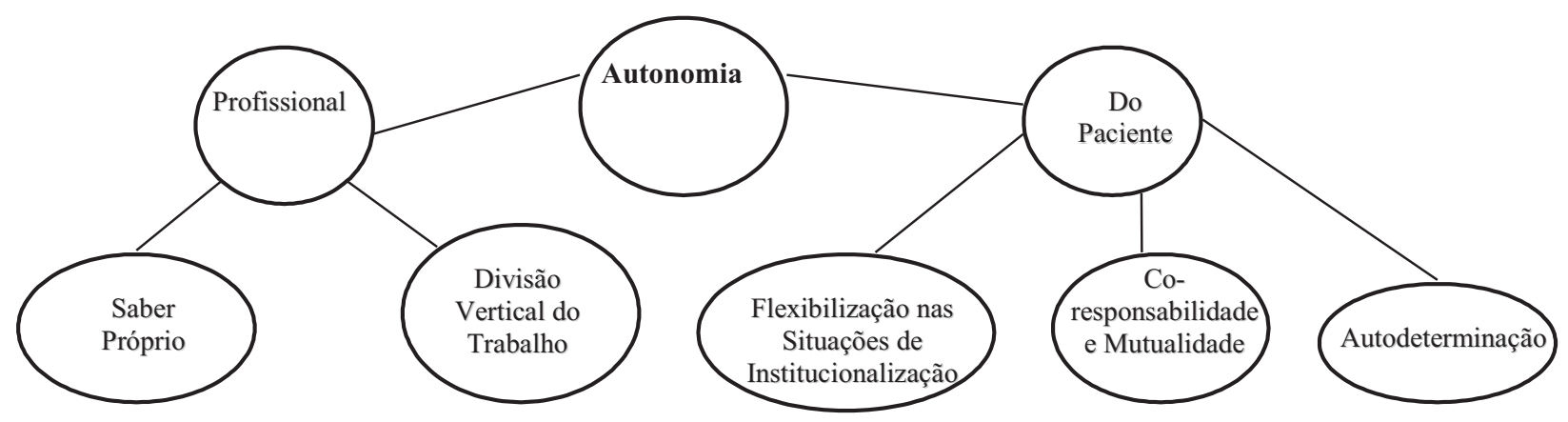

Figura 1 - Vertentes encontradas e suas categorias dentro do tema autonomia

Tema: Autonomia; Vertente: Autonomia profissional; Categoria: Saber próprio.

O saber próprio da enfermagem é considerado como um mosaico, na medida em que vários conhecimentos são considerados como constituintes da profissão. Ao mesmo tempo, é importante para a autonomia profissional a constituição de um saber específico que tem como significado a delimitação da essencialidade da profissão e a constituição de um espaço próprio de poder ${ }^{(1)}$.

Os conhecimentos consubstanciados nas teorias de enfermagem são considerados como próprios da profissão, assim como a consulta de enfermagem, a educação em saúde e a sistematização da assistência de enfermagem. As teorias orientam a prática descrevendo, explicando ou prevendo os fenômenos. Segundo um estudo, a teoria é definida como um conjunto de conceitos inter-relacionados, definições e proposições que apresentam uma forma sistemática de ver os fatos/eventos, pela especificação das relações entre as variáveis, com a finalidade de prever o fato/evento ${ }^{(2)}$.

A consulta de enfermagem é vista como uma estratégia que possibilita a integralidade de atenção à saúde. Pressupõe as seguintes fases: histórico de enfermagem (obtido através da entrevista e do exame físico); diagnóstico de enfermagem (análise e julgamento das informações); prescrição de enfermagem e evolução de enfermagem.

A assistência de enfermagem sistematizada é a forma do profissional organizar e inter-relacionar suas ações, permitindo-lhe dirigir e controlar seu próprio trabalho, de modo a atingir metas definidas de comum acordo com a clientela ${ }^{(3)}$.

Tema: Autonomia; Vertente: Autonomia profissional; Categoria: Divisão vertical do trabalho.

A profissão enfermagem foi constituída historicamente em complementaridade do trabalho médico. Havendo, dessa forma, uma divisão vertical do trabalho, no qual se se- para a concepção (intelectual) da execução (manual). Essa mesma relação ocorre dentro da enfermagem, cabendo à enfermeira o trabalho mais intelectual, e aos trabalhadores de enfermagem de nível médio, o trabalho mais manual e supervisionado pela mesma ${ }^{(4)}$. Por conseguinte, os serviços de saúde são moldados de acordo com o modelo assistencial clínico, cuja finalidade é a recuperação do corpo individual e o instrumento essencial de trabalho é o saber anátomofisiológico $^{(4)}$.

Outro ponto relevante é que o enfermeiro investe o maior tempo de seu trabalho resolvendo questões administrativas da assistência. Este fazer gera um falso reconhecimento e uma falsa autonomia por parte do mesmo, uma vez que não se estabelecem vínculos com o paciente e, deixam de conferir aos enfermeiros, pela ausência de vínculos, um certo poder instituído ${ }^{(5)}$.

Quanto à autonomia do paciente foram encontradas as categorias temáticas: co-responsabilidade/mutualidade, flexibilização nas situações de institucionalização e autodeterminação.

Tema: Autonomia; Vertente: Autonomia do paciente; Categoria: Flexibilização nas situações de institucionalização.

Diante do desconhecido e, sobretudo, diante da dor e das limitações físicas, é uma tendência do ser humano reagir com algum grau de regressão. Os profissionais de saúde tendem a reforçar ainda mais a regressão do paciente, já que estes aceitam docilmente as normas e rotinas impostas pela instituição. Desse modo, o hospital contemporâneo transforma o doente em um mero objeto de cuidados $^{(4,6-7)}$.

De acordo com o próprio surgimento histórico da enfermagem dentro dos hospitais, num regime disciplinante, a prática de enfermagem vê-se voltada para as normas e rotinas impostas, visando o controle dos corpos doentes, trabalhando de uma forma paternalista. Esta atitude é justifi- 
cada pelo conhecimento científico que o profissional detém e que seus assistidos não detém, restando ao paciente a posição de recebedor de um bem feito por outro ${ }^{(5,7,8-10)}$.

A informação prestada ao paciente limita-se ao que o profissional julga ser relevante, muitas vezes não incorporando a compreensão que o mesmo tem sobre sua doença, os valores pessoais, seus medos e receios ${ }^{(8)}$. Há práticas de educação em saúde pautadas na prescrição de normas e condutas, servindo mais a sujeição do cliente do que a sua libertação ${ }^{(6)}$. Os hábitos de saúde e a concepção da sociedade quanto à cura de doenças tornam-se controlados pelas determinadas visões hegemônicas, legitimadas como as únicas eficazes.

Como consequiência desse fato, o cidadão vê-se expropriado da capacidade de executar comportamentos autônomos em relação à sua saúde, vivendo subordinado a inúmeras regras de comportamento ${ }^{(11)}$.

Há, portanto, uma correlação do processo de autonomização do paciente com as práticas realizadas pelos trabalhadores de saúde e destas com o grau de flexibilização das instituições.

\section{Tema: Autonomia; Vertente: Autonomia do paciente; Categoria: Autodeterminação.}

A autonomia pode ser definida como habilidade de autodeterminação, de ser independente, isto é, o indivíduo tem o poder e a habilidade de decidir ou agir sobre si próprio ${ }^{(3,9)}$. Dessa forma, é importante que na sistematização da assistência de enfermagem o usuário passe de objeto para sujeito da assistência.

Para tanto, é importante a função educativa do enfermeiro numa postura ética, que visualize o usuário como capaz de ação própria e resolutiva para a solução de seus problemas, contribuindo, dessa forma, para a desalienação e libertação desse sujeito. O usuário poderá experimentar um poder de decisão maior sobre seu próprio corpo, com base em conhecimentos compartilhados sobre sua saúde ${ }^{(6,12)}$.

Na perspectiva da Saúde Coletiva, evidencia-se o modelo radical da educação em saúde, que é centrado no método de conscientização crítica dos indivíduos, com o objeti- vo promover a saúde a partir de uma vida vivida com qualidade. Trabalha com grupos ao invés de alvos isolados, buscando mais a mudança social do que somente a transformação pessoal. Tem a intenção de promover o envolvimento dos indivíduos na tomada de decisões relacionadas a sua própria saúde e ao grupo social a que pertencem ${ }^{(12)}$. Este tipo de modelo busca a construção de uma comunidade cada vez mais ativa e empowered, ou seja, que possui autonomia para fazer escolhas informadas ${ }^{(12)}$.

Nessa perspectiva, o cuidado domiciliar é uma estratégia que identifica as necessidades sociais e de saúde da população. Esse contexto enfatiza a autonomia do paciente e esforça-se em aumentar e realçar suas habilidades do seu ambiente $^{(13)}$.

\section{Tema: Autonomia; Vertente: Autonomia do paciente; Categoria: Co-responsabilidadel Mutualidade}

A mutualidade é definida como associação e compartilhamento entre as autonomias profissional e do paciente, proporcionando, dessa forma, benefícios e contribuição de ambos para o processo de cuidado à saúde ${ }^{(9)}$. A ênfase está no pressuposto de que o cliente é um ser humano único, com suas peculiaridades e potencialidades de crescimento, através de um relacionamento de ajuda, que propicia ao sujeito a oportunidade de realizar escolhas conscientes e responsáveis ${ }^{(9,14)}$.

As autonomias do profissional e do sujeito devem estar em interação dinâmica. Quando a situação vivenciada exigir conhecimentos e procedimentos técnico-científicos, a autonomia profissional será o foco principal. Em outros, o foco recairá sobre o sujeito, que demonstrará as suas peculiaridades, as suas necessidades e o seu querer. Da interação das duas autonomias resultará a mutualidade do cuidado ${ }^{(9)}$.

Quanto à temática necessidades de saúde, também foram encontradas duas vertentes de análise: necessidades biológicas $^{(3)}$ e necessidades sociais ${ }^{(2)}$.

As vertentes do tema necessidades de saúde que são as necessidades biológicas e as necessidades sociais interceptam-se com as necessidades psicológicas para dar formato às necessidades biopsicossociais, ilustradas na Figura 2.

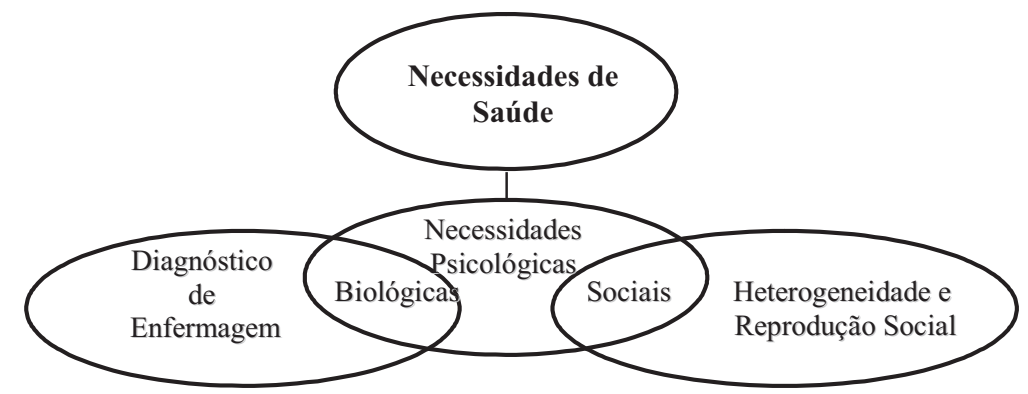

Figura 2 - Vertentes encontradas sobre a temática necessidades de saúde 
Tema: Necessidades de Saúde; Vertente: Necessidades biológicas.

$\mathrm{Na}$ literatura estudada, encontrou-se uma freqüência maior de trabalhos que reduziam o objeto do cuidado às necessidades exclusivamente biológicas. A própria definição de diagnóstico de enfermagem que é o julgamento clínico das respostas do indivíduo, da família ou da comunidade aos processos vitais ou aos problemas de saúde reais ou potenciais $^{(15)}$, transmite a visão biologicista do cuidado.

\section{Tema: Necessidades; Vertente: Necessidades Sociais.}

Outra vertente para o tema necessidades de saúde é o que amplia a concepção de necessidades, para compreender suas raízes e define que as necessidades não são homogêneas e que a heterogeneidade das necessidades é definida pela inserção de classe dos indivíduos ${ }^{(16)}$. Nesta perspectiva, necessidades de saúde são necessidades de reprodução social, ou seja, são necessidades de indivíduos das diferentes classes, são diferentes nos diferentes grupos sociais, definidos pela inserção na divisão social do trabalho, determinante dos diferentes modos de viver ${ }^{(16)}$.

Em razão dessa origem social, produzem-se necessidades que correspondem a de muitas pessoas e, potencialmente, de todas, a depender de seu grupo social ${ }^{(17)}$. Assim sendo, para atender necessidades de saúde de uma dada população, é necessário instaurar diferentes práticas, que respondam a essas necessidades.

Esse tema é complexo, já que pelo modo como os serviços de saúde são organizados, há uma oferta de ações, a partir de demanda, sem que se considere as necessidades como um todo.

Tomar as necessidades de saúde como objeto das práticas implica em considerar a inserção dos indivíduos nos diferentes grupos sociais, dessa forma, as estratégias em saúde irão abranger,

além da dimensão biológica, as dimensões: cultural, econômica, ecológica e política, que são comandadas no modo de produção capitalista - pela dimensão econômica ${ }^{(16)}$.

\section{Tema: Sistematização da Assistência de Enfermagem}

A sistematização da assistência se faz necessária para a avaliação crítica da pertinência e relevância do trabalho de enfermagem frente ao atendimento das necessidades de saúde. Busca-se tomar como objeto do processo de trabalho em saúde, as necessidades e intervenções específicas da profissão, na assistência e no cuidado dos indivíduos, famílias e grupos sociais.

No Brasil, pode-se utilizar como ferramenta um projeto de classificação em construção que é o CIPESC (Classificação Internacional das Práticas de Enfermagem em Saúde
Coletiva), inspirado no CIPE (Classificação Internacional das Práticas de Enfermagem), cuja origem deu-se no CIE (Conselho Internacional de Enfermeiros). O projeto CIPESC configura-se como o pioneiro ao demonstrar o que faz a enfermagem nos serviços básicos de saúde e analisar o porque deste fazer. No entanto, sua aplicabilidade, assim como a estruturação da sistematização da assistência, depende do envolvimento do profissional na prática de enfermagem, capaz de prover cuidados para transformar e solucionar os problemas das pessoas e da coletividade.

Os sistemas NANDA, NIC e NOC são relacionados às necessidades biológicas; uma vez que a NANDA nomeia as necessidades de cuidado de enfermagem a partir de sinais e sintomas, a NIC propõe as intervenções de cuidado necessárias encontradas a partir do diagnóstico de enfermagem e a NOC avalia a eficácia desse cuidado. Evidencia-se nestes sistemas a preocupação com a autonomia profissional, pois estes traçam o caminho para o cuidado e, dessa forma, as ações de enfermagem podem ser comparadas, inclusive por diversos países que utilizam tais sistemas, favorecendo com que a prática de enfermagem seja reconhecida e padronizada.

Os projetos CIPESC e CIPE aproximam-se mais das necessidades biopsicossociais. O CIPESC busca padronizar a linguagem em atenção básica à saúde, esta é moldada de acordo com a ênfase maior do cuidado, na perspectiva de Saúde Coletiva. O projeto CIPE é desenvolvido como um marco unificador das diferentes classificações, portanto não apresenta parte de uma visão unilateral do processo saúdedoença, mas sim unifica todas as formas de conhecimento. Portanto, o CIPE e o CIPESC aproximam-se mais da autonomia do paciente, uma vez que buscam o reconhecimento da sua forma de viver, propiciando estratégias que fortalecem a autonomia dos assistidos. Também são instrumentos para aumentar a visibilidade e o reconhecimento profissional, promovendo maior autonomia para a enfermagem.

\section{CONCLUSÃO}

A sistematização da assistência de enfermagem moldada de acordo com o novo paradigma de atenção à saúde deve se inter-relacionar com a autonomia profissional, autonomia do paciente, necessidades biológicas e necessidades sociais da população assistida.

No que tange à autonomia profissional, destaca-se a assistência de acordo com as teorias de enfermagem, a consulta de enfermagem e a estruturação dos serviços de saúde como oportunizadores da autonomia do enfermeiro, assim como a inserção efetiva do enfermeiro no processo de trabalho em saúde e a politicidade profissional como fatores que promovem a autonomização.

Quanto à autonomia do paciente, esta deve ser uma premissa na assistência, para tanto é necessário atividades que 
fortaleçam o empowerment, este favorece a autodeterminação e a independência do mesmo na prevenção, promoção e recuperação de sua saúde. Uma estratégia que favorece a autonomia do paciente é o cuidado domiciliar, pois há atendimento no âmbito social no qual está inserido e do qual depende. Dessa forma, a educação, tratamento e prevenção em saúde adequam-se ao modo de vida.

Sob a perspectiva da Saúde Coletiva as necessidades biológicas são importantes, mas devem estar articuladas as

\section{REFERÊNCIAS}

1. Gomes AMT, Oliveira DC. A representação social da autonomia profissional do enfermeiro na Saúde Pública. Rev Bras Enferm. 2005;58(4):387-92.

2. George JB. Teorias de enfermagem: dos fundamentos à prática profissional. $4^{\mathrm{a}}$ ed. Porto Alegre: Artes Médicas; 2000.

3. Nietsche EA, Backes VMS. A autonomia como um dos componentes básicos para o processo emancipatório do profissional enfermeiro. Texto Contexto Enferm. 2000; 9(3):153-74.

4. Kohlrausch E. O modelo assistencial clínico e algumas possibilidades de fazer diferente. Rev Gaúcha Enferm. 1999;20 (n.esp):70-85 .

5. Boemer MR, Sampaio MA. O exercício da enfermagem em sua dimensão bioética. Rev Lat Am Enferm. 1997;5(2):33-8.

6. Wendhhausen A. Assistência de enfermagem: da sujeição dos copos à autonomia do sujeito. Cogitare Enferm. 1997;2(1):21-4.

7. Martin LM. A ética e a humanização hospitalar. Mundo Saúde. 2003;27(2):206-18.

8. Barros VL, Fortes PAC. A informação esclarecedora e o consentimento esclarecido: um desafio ao Programa de Saúde da Família na garantia dos direitos do paciente. Mundo Saúde 2003; 27(2):280-91.

9. Sadala MLA. Autonomia/mutualidade na assistência de enfermagem. Rev Lat Am Enferm. 1996;4(1):111-17. necessidades sociais, portanto, é necessário sistematizar o cuidado a partir dessas, que são heterogêneas e têm suas origens na reprodução da vida em sociedade. No entanto, as necessidades ainda são vistas preponderantemente numa perspectiva biológica.

De acordo com os achados, evidencia-se a necessidade de maiores estudos que considerem a autonomia e as necessidades de saúde no processo de sistematização da assistência de enfermagem.

10. Pires MRGM. Politicidade do cuidado como referência emancipatória para a enfermagem: conhecer para cuidar melhor, cuidar para confrontar, cuidar para emancipar. Rev Lat Am Enferm. 2005;13(5):729-36.

11. Perna PO. Saúde, autonomia e trabalho. Cogitare Enferm. 1996;1(1):52-9.

12. Oliveira DL. A "nova" saúde pública e a promoção da saúde via educação: entre a tradição e a inovação. Rev Lat Am Enferm. 2005;13(3):423-31.

13. Paz AA, Santos BRL. Programas de Cuidado de Enfermagem Domiciliar. Rev Bras Enferm. 2003;56(5):538-41.

14. Fanes L, Maftum MA. Consulta de enfermagem sustentada na ralação de ajuda: a prática assistencial à pessoa portadora do Mal de Chagas. Cogitare Enferm. 2002; 7(2):10-20.

15. Pasini D, Alvim I, Kanda L, MendesSP, Cruz DALM. Diagnósticos de enfermagem de pacientes em Unidades de Terapia Intensiva. Rev Esc Enferm USP. 1996;30(3): 501-18.

16. Campos CMS, Mishima SM. Necessidades de Saúde pela voz da sociedade civil e do Estado. Cad Saúde Pública 2005; 21(4):1260-8.

17. Schraiber LB, Mendes-Gonçalves RB. Necessidades de saúde e atenção primária. In: Schraiber LB, Nemes MIB, MendesGonçalves RB. Saúde do adulto: programas e ações na unidade básica. $2^{a}$ ed. São Paulo: Hucitec; 2000. p. 29-47. 